\title{
Lifestyle behaviours associated with diet quality in higher education students: a systematic review
}

\author{
S. Doak ${ }^{1}$, N. O’Callaghan ${ }^{1}$, J. Kearney ${ }^{2}$, J.M. McCormack ${ }^{1}$ and L. Keaver ${ }^{1}$ \\ ${ }^{1}$ Department of Health and Nutritional Science, Institute of Technology Sligo, Ash Lane, Sligo, Ireland and \\ ${ }^{2}$ School of Biological and Health Sciences, Technological University Dublin, City Campus, Kevin Street, Dublin, \\ Ireland
}

Evidence suggests that unhealthy diet and lifestyle behaviours correlate with each other, causing an increase in co-morbidities, obesity and affecting overall health ${ }^{(1)}$. During the transition from secondary school into higher education many lifelong health-related behaviours are established ${ }^{(2)}$. If identified early, modifying health behaviours can reduce the disease risk of students in later life ${ }^{(3)}$. The aim of this review is to identify the relationship between dietary quality and lifestyle behaviours in higher education students.

A systematic search was performed in the databases PubMed, Web of Science, EOLAS, ScienceDirect, and Eric in accordance with PRISMA guidelines. Inclusion criteria were studies conducted among higher education students, dietary intake assessment and its association with a lifestyle behaviour, use of validated tools, and published in English from 2000-2021. One reviewer assessed the title/ abstract, and two reviewers independently assessed the full papers. Data were extracted, and quality assessment was conducted using the Academy of Nutrition and Dietetics Quality Criteria Checklist tool.

Thirty-six papers with a total of 173,416 individuals met the eligibility criteria and were included. Studies were from a total of thirty-eight countries in six continents, with the highest amount in the USA $(n=10)$ and Spain $(n=10)$ and none from Ireland or the UK. Causal relationships could not be established as all, but three studies had a cross-sectional design. Three dietary categories were developed: i) diet quality $(n=6)$, assessed by dietary indices, (e.g., Healthy Eating Index) ii) dietary patterns $(n=11)$, assessed by the Mediterranean diet (e.g., The Mediterranean Diet Quality Index), and iii) fruit and vegetable consumption ( $=19)$, assessed by servings (e.g., How many servings of fruits and vegetables do you usually have per day?). Lifestyle behaviours included: physical activity $(n=21)$, sleep $(n=6)$, alcohol $(n=5)$, smoking $(n=17)$, and 'other' substance use $(n=4) .16$ of 21 studies $(76 \%)$ found a significant relationship between higher diet quality and higher physical activity levels. Higher alcohol use was associated with low intake of fruit $(\mathrm{n}=5,100 \%)$ and vegetables $(\mathrm{n}=4,80 \%)$ consumption. 8 of the 17 studies $(47 \%)$ that tested smoking status and diet found a significant relationship between poor diet quality and smoking. Only 1 of $6(17 \%)$ papers found a significant association between better sleep quality and diet. One paper found that higher fruit and vegetable intake was associated with increased marijuana use among females, whereas no significant association was found between diet scores and any 'other' substance use $(\mathrm{n}=3)$.

There is evidence of a correlation between higher physical activity levels, lower alcohol consumption and higher diet quality. Smoking status appears to be more inconclusive, whereas sleep, 'other' substance use and diet do not appear to have a relationship. Additional research and clarity of the relationship between diet and lifestyle behaviours are warranted in this population group.

\section{References}

1. Gherasim A, Arhire LI, Nită O, et al. (2020) Proc Nutr Soc 79(3), 311-323.

2. Pachucki MA (2011) Int J'Obes 36(5), 686-694.

3. Gall SL, Jamrozik K, Blizzard L, et al. (2009) Eur J Cardiovasc Prev Rehabil 16(6), 684-689. 